\title{
Nephrologischer Arbeitskreis Saar-Pfalz-Mosel e. V. 27. Wissenschaftliches und Pflegesymposium am 02.07.2016
}

Zum 27. Wissenschaftlichen und Pflegesymposium des Nephrologischen Arbeitskreises Saar-Pfalz-Mosel e. V. möchten wir Sie herzlich in das Tagungszentrum im Logenturm Ost im Fritz-Walter-Stadion in Kaiserslautern einladen.

Der Nephrologische Arbeitskreis, eine Fachgesellschaft niedergelassener und klinisch tätiger Nephrologen, besteht seit über 30 Jahren. Wir haben es uns zur Aufgabe gemacht, einmal jährlich in einer Tagung umfassende aktuelle Informationen zu Nierenerkrankungen und deren Behandlung zu geben. Da bei der Behandlung nierenerkrankter Patienten unterschiedliche Arzt- und Berufsgruppen beteiligt sind und die berufsgruppenübergreifende $\mathrm{Zu}$ sammenarbeit besonders wichtig ist, veranstaltet der Arbeitskreis seit 2007 ein gemeinsames ärztliches und pflegerisches Symposium, auch mit gemeinsamen Sitzungen von Ärzten und Pflegekräften. Die Themenschwerpunkte sind deshalb natürlich eher in der Medizin, aber in der Pflege auch in der Berufspolitik.
Die AfnP ist nicht Mitveranstalter des Symposiums. Sie unterstützt aktiv den Nephrologischen Arbeitskreis v.a. in den pflegerisch ausgerichteten Teilen des Symposiums. Aus diesem Grund können AfnP-Mitglieder auch nicht ihre Bildungsgutscheine bei diesen Veranstaltungen einlösen; sie müssen den vollen, allerdings günstigen Eintrittspreis an den Veranstalter, den Arbeitskreis Saar-PfalzMosel e.V., entrichten.

\section{Einladung und Programm}

Für die Vorträge in den Updates und den Symposien konnten wir wie gewohnt renommierte Referenten gewinnen. Hervorheben möchten wir die Teilnahme von Dr. Markus Mai, Trier, der als erster Präsident der Pflegekammer Rheinland-Pfalz ein Statement zum aktuellen Stand der Pflegekammer geben wird. Begleitet wird das Symposium wie jedes Jahr von einer Industrieausstellung, auf der die Besucher sich über medizinische und technische Innovationen in der Nephrologie informieren können.

Nach dem Ende des Symposiums gegen 18:00 Uhr findet ab 18:30 Uhr noch eine kostenlose Stadionführung statt. Um eine Voranmeldung wird hierbei gebeten, um die Gruppengröße etwas besser einschätzen zu können. Über die Tagung hinaus bietet Kaiserslautern zahlreiche attraktive Ausflugsziele sowie ein reiches kulturelles Programm. Nähere Informationen finden Sie unter www.kaiserslautern.de. Wir würden uns sehr freuen, Sie zu unserer Veranstaltung in Kaiserslautern begrüßen zu dürfen.

Für den Nephrologischen Arbeitskreis e. V.: T. Rath, M. Marx, S.M. Weiner, K. Frommherz Für die Arbeitsgemeinschaft für nephrologisches Personal e. V. (AfnP):

M. Breit, R. Nicklas

\section{Programm 27. Wissenschaftliches und Pflegesymposium in Kaiserslautern (Samstag, der 02.07.2016)}

08:30 Uhr Registrierung und Eröffnung

Updates I (Ärzte und Pflege)

Vorsitz: Dr. T. Ruf, Kaiserslautern

9:00 Uhr Update Klinische Nephrologie Prof. Dr. S. Weiner, Trier

9:30 Uhr Update Transplantation Dr. T. Rath, Kaiserslautern

10:00 Uhr Update Hämodialyse Prof. Dr. G. Heine, Homburg

10:30 Uhr Pause - Industrieausstellung

Symposium I (Ärzte)

Vorsitz: N.N.

11:00 Uhr Nephropathie bei Gammopathien Prof. Dr. Lorenz Sellin, Düsseldorf

11:30 Uhr Nierenbiopsie-Konferenz Prof. Dr. R. Waldherr, Heidelberg

Symposium II (Parallelprogramm Pflege)

Vorsitz: $\quad$ M. Breit, Trier

11:00 Uhr 1/2 Jahr Pflegekammer Rheinland-Pfalz

Dr. rer. cur. M. Mai, Trier

11:30 Uhr Hygiene; neue Leitlinie in der Dialyse I M. Neumann, Trier

12:00 Uhr Hygiene; neue Leitlinie in der Dialyse II M. Neumann, Trier

12:30 Uhr Mittagspause - Industrieausstellung

Forum der Industrie (Ärzte)

Vorsitz: N.N.

13:45 Uhr Therapie der ADPKD PD Dr. R.-U. Müller, Köln
14:15 Uhr Hypercholesterinämie Dr. A. Wagner, Trier

14:45 Uhr Immunadsorption V. Klein

15:15 Uhr Hepatitis-C-Therapie Dr. A. Rieke

Symposium III (Parallelprogramm Pflege)

Vorsitz: $\quad$ A. Leidinger, Wadgassen

13:45 Uhr Grundlagen der Nierenersatztherapien M. Breit, Trier

14:15 Uhr Immunologie und Immunsuppression R. Barrientos

15:00 Uhr NTx, Besonderheiten der AB0- inkompatiblen NTx

Dr. S. Ziefle, Kaiserslautern

15:45 Uhr Pause - Industrieausstellung

Updates II (Ärzte und Pflege)

Vorsitz: N.N.

16:15 Uhr Update Intensivmedizin Fr. Dr. Benner

16:45 Uhr Update Peritonealdialyse Dr. N. Wirtz

17:15 Uhr Update Hypertonie PD Dr. M. Marx, Völklingen

17:45 Uhr Fazit/Verabschiedung, Ausblick 2017

T. Rath, M. Breit

18:00 Uhr Ende

Veranstaltungsort: Logenturm Ost im Fritz-Walter-Stadion (FCK Gastronomie GmbH),

Fritz-Walter-Straße 1, 67663 Kaiserslautern

Termin: Samstag, 02. Juli 2016

Veranstalter: Nephrologischer Arbeitskreis Saar-Pfalz-Mosel e. V. in Kooperation mit der AfnP (Arbeitsgemeinschaft für nephrologisches Personal)

Gebühren Symposium:

Chefärzte, Oberärzte, Fachärzte: 30 Euro $\quad$ Assistenzärzte, Pflegepersonal: 15 Euro Studenten, Auszubildende: 0 Euro

Information - Anmeldung - Registrierung - Organisation - Industrieausstellung: Julia Nesselrath, Transplantationsadministration, Westpfalz-Klinikum GmbH, Hellmut-HartertStr. 1, 67655 Kaiserslautern, Tel.: (+49631) 203-1693, Fax: (+49631) 203-1647, E-Mail: jnesselrath@westpfalz-klinikum.de

Die CME-Zertifizierung bei der Ärztekammer Rheinland-Pfalz sowie Fortbildungspunkte für die Pflege sind beantragt.

Für weitere Details siehe Flyer, der dieser Ausgabe der Dialyse aktuell beigelegt ist. 